\title{
The Apparent Madness of Crowds: Irrational collective behavior emerging from interactions among rational agents
}

\author{
Sitabhra Sinha \\ The Institute of Mathematical Sciences, C. I. T. Campus, Taramani, \\ Chennai - 600 113, India. \\ sitabhra@imsc.res.in \\ Standard economic theory assumes that agents in markets behave rationally. \\ However, the observation of extremely large fluctuations in the price of fi- \\ nancial assets that are not correlated to changes in their fundamental value, \\ as well as the extreme instance of financial bubbles and crashes, imply that \\ markets (at least occasionally) do display irrational behavior. In this paper, \\ we briefly outline our recent work demonstrating that a market with interact- \\ ing agents having bounded rationality can display price fluctuations that are \\ quantitatively similar to those seen in real markets.
}

\section{Introduction}

It has long been debated in the economic literature whether markets exhibit irrational behavior [1. The historical observations of apparent financial "bubbles", in which the demand (and therefore, the price) for certain assets rises to unreasonably high levels within a very short time only to come crashing down later 2], imply that markets act irrationally, because the rapid price changes are not associated with changes in the fundamental value of the assets. Believers in rational expectation theory argue that the price rise actually reflects the market's expectations about the long-term prospect of these assets and the large fluctuations are just rapid adjustments of these expectations in the light of new information 3. These advocates of the "efficient market" school of thought claim that popular descriptions of speculative mania (e.g., in Ref. 4) have been often exaggerated. However, critics point out that the market's estimate of the long-term value of an asset is a quantity that cannot be measured, and therefore, it is difficult to verify whether historical bubbles were indeed rational outcomes.

In this paper, we take an intermediate position between these two opposing camps. We assume that individual agents do behave in a rational manner, where rationality is identified with actions conducive to market equilibrium. 
In other words, rational agents will act in such a way that the market is "balanced", exhibiting neither excess demand nor supply. Therefore, we expect only small fluctuations about the equilibrium when we have a large ensemble of non-interacting agents. In the model presented in this paper, market behavior is described by the collective decision of many interacting agents, each of whom choose whether to buy or sell an asset based on the limited information available to them about its prospects. In isolation, each agent behaves so as to drive the market to equilibrium. We investigate the possibility that interactions between such agents can severely destabilize the market equilibrium. In fact, we show that when agents are allowed to modify their interactions with neighbours, based on information about their past performance in the market, this results in the market becoming unbalanced and exhibiting extremely large fluctuations that are quantitatively similar to those seen in real markets.

\section{Collective irrationality in an agent-based model}

In this section, we present an agent-based model of the fluctuation of demand for a particular asset. The agents are assumed to be operating under bounded rationality, i.e., they try to choose between buying and selling the asset based on information about the action of their immediate neighbors and how successful their previous choices were. The fundamental value of the asset is assumed to be unchanged throughout the period. From the "efficient markets" hypothesis, we should therefore expect to see only small departures from the equilibrium. In addition, the agents are assumed to have limited resources, so that they cannot continue to buy or sell indefinitely. However, instead of introducing explicit budget constraints [5], we have implemented gradually diminishing returns for a decision that is taken repeatedly.

We assume that all agents are placed on a lattice, each site being occupied by one agent. An agent can only interact with its immediate neighbors on the lattice. In the simulations reported here, we have considered a two-dimensional hexagonal lattice, so that the number of neighbors is $z=6$. At any given time $t$, the state of an agent $i$ is fully described by two variables: its choice, $S_{i}^{t}$, and its belief about the outcome of the choice, $\theta_{i}^{t}$. The choice can be either buy $(=+1)$ or sell $(=-1)$, while the belief can vary continuously over a range. The behavior of the agent over time can then be described by the equations governing the dynamics of $S$ and $\theta$,

$$
S_{i}^{t+1}=\operatorname{sign}\left(\Sigma_{j} J_{i j}^{t} S_{j}^{t}-\theta_{i}^{t}\right), \theta_{i}^{t+1}=\theta_{i}^{t}+\mu_{i} S_{i}^{t+1},
$$

where, $J_{i j}^{t}$ measures the degree of interaction between neighboring agents. The adaptation rate, $\mu_{i}$, governs the time-scale of diminishing returns, over which the agent switches from one choice to another in the absence of any interactions between agents. The overall state of the market at any given time is described by the fractional excess demand, $M^{t}=(1 / N) \Sigma_{j} S_{j}^{t}$.

In previous work [6], we have shown that, if the interactions between agents do not change over time (i.e., $J_{i j}^{t}=J$, a constant), then $M$ shows only small 

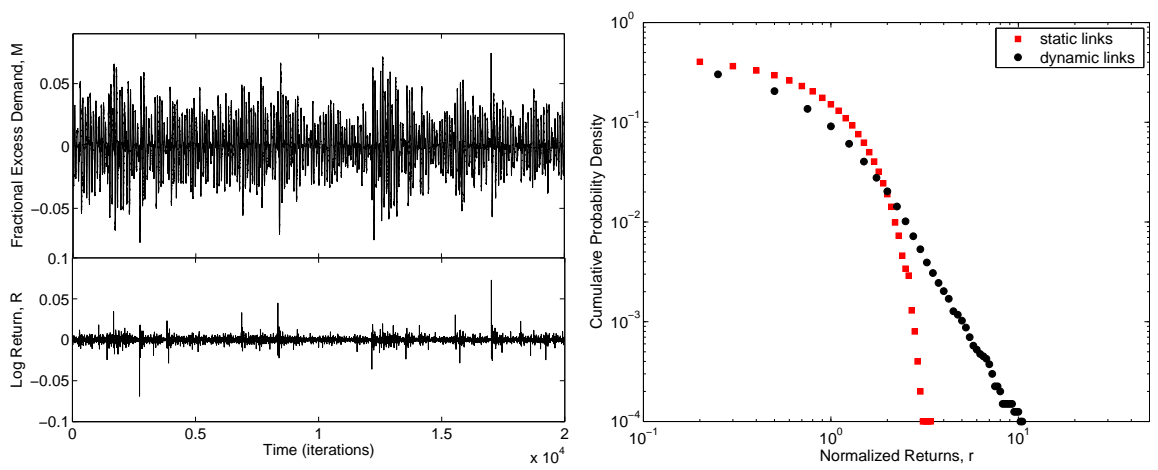

Fig. 1. Spontaneous fluctuations through dynamic interactions in a system of $400 \times 400$ agents. The adaptation rates, $\mu_{i}$, are chosen from an uniform random distribution between $[0,0.1]$. (Left) The time series of the fractional excess demand, $M$ (top) and the corresponding logarithmic return, $R$ (bottom), exhibiting significant number of large fluctuations. (Right) The cumulative probability density function of normalized returns, $r$, comparing the case where the $J_{i j}$ are fixed in time (squares) with the situation when the $J_{i j}$ change over time (circles). The latter shows a power-law decay with an exponent close to 3 .

fluctuations about 0 . This accords with the "efficient markets" hypothesis that any transient imbalance in the demand or supply of the asset is quickly corrected through the appropriate response of agents, so that the market remains more or less in equilibrium. However, if the agents have access to global information about the market (i.e., $M$ ), under certain conditions this can lead to large deviations from the market equilibrium. We have previously shown that if $M$ is allowed to affect the belief dynamics of agents, then the market spends most of the time in states corresponding to excess demand or excess supply. This kind of two-phase behavior [7] points to the destabilizing effect of apparently innocuous information exchanges in the market.

Very recently, we have observed that the collective behavior can also be destabilized if, instead of affecting the belief dynamics, the knowledge of $M$ is used in evolving the structure of interactions $J_{i j}^{t}$ between neighboring agents. This is implemented by assuming that agents seek out the most successful agents in its neighborhood, and choose to be influenced by them preferentially. Here, success is measured by the fraction of time the agent's decision (to buy or sell) accorded with the market behavior. As a rise in excess demand of an asset is taken to signal its desirability, an agent is considered successful if it is in possession of an asset that is in high demand. If an agent $i$ is successful in predicting the market (i.e., its action in the last round accorded with the majority decision of the collective) then its interaction structure is unchanged. Otherwise, its neighboring agents with higher success are identified and the link strength between them, $J_{i j}$, is adjusted by an amount that is proportional to the ratio of the success of agent $j$ to agent $i$. This implies that agents with 
higher success affect the decision process of agents with less success, but not the other way around. Finally, $J_{i j}$ is normalized such that, for each agent, $\Sigma_{i} J_{i j}=1$.

Fig. 1(left) shows the resulting time series of the fractional excess demand. As the price $P$ of the asset is governed by the demand for it, we can take $P$ to be linearly related to $M$. This allows us to quantify the price fluctuations for the asset by calculating the logarithmic return of $P$ as $R^{t}=\ln P^{t+1}-$ $\ln P^{t}$. It is evident that the fluctuations are much larger than what would have been expected from an uncorrelated random process. This is further established when we plot the distribution of the return, normalized by its standard deviation, and compare it with the case where the $J_{i j}$ are constant in time (Fig. 1 right). While the latter case is consistent with a Gaussian distribution, the model with adaptive interaction dynamics is found to exhibit a return distribution that has a power law tail. Moreover, the exponent of the cumulative distribution, $\alpha \simeq 3$, is found to agree quantitatively with the corresponding values observed in actual markets 8 .

\section{Conclusions}

The observation of large price fluctuations (most strikingly during bubbles or crashes) implies that markets often display instabilities where the demand and supply are not even approximately balanced. We have seen in this paper that this is not necessarily inconsistent with the assumption that individual economic agents are rational. A simple agent-based model, where the structure of interactions between agents evolve over time based on information about the market, exhibits extremely large fluctuations around the market equilibrium that qualitatively match the fluctuation distribution seen in real markets.

\section{References}

1. Albin P S (1998) Barriers and bounds to rationality. Princeton University Press, Princeton

2. Chancellor E (1999) Devil take the hindmost: A history of financial speculation. Macmillan, London

3. Garber P M (1990) Famous first bubbles, J. Economic Perspectives 4:35-54

4. MacKay C (1852) Memoirs of extraordinary popular delusions and the madness of crowds. National Illustrated Library, London

5. Iori G (2002) A microsimulation of traders activity in the stock market: the role of heterogeneity, agents' interaction and trade frictions, J. Economic Behavior \& Organization 49:269-285

6. Sinha S, Raghavendra S (2004) Phase transition and pattern formation in a model of collective choice dynamics, SFI Working Paper 04-09-028

7. Sinha S, Raghavendra S (2005) Emergence of two-phase behavior in markets through interaction and learning in agents with bounded rationality. In: Takayasu H (ed) Practical fruits of econophysics. Springer, Tokyo :200-204

8. Gopikrishnan P, Meyer M, Amaral L A N, Stanley H E (1998) Inverse cubic law for the distribution of stock price variations, Eur. Phys. J. B 3:139-140 